\title{
Ciencia y Fútbol
}

Pensar en Movimiento:

Revista de Ciencias del Ejercicio y la Salud

ISSN 1659-4436

Vol. 15, No.1, pp. 12- 22

Cierre al 30 de junio, 2017

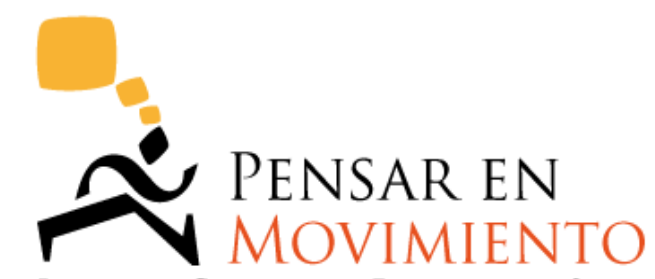

REVISTA DE CIENCIAS DEL EJERCICIO Y LA SALUd

\section{DEMANDAS CINEMÁTICAS EN DOS TIPOS DE ESPACIOS REDUCIDOS EN JUGADORES UNIVERSITARIOS DE FÚTBOL DE COSTA RICA ${ }^{1}$}

\author{
Daniel Rojas-Valverde, M.Sc. ${ }^{2,3}(A, B, C, D, E)$; María Morera-Castro, Ph.D. ${ }^{3(C, D, E)}$; Jaqueline \\ Montoya-Rodríguez ${ }^{2,3(B, C, D, E)}$; y Randall Gutiérrez-Vargas, M.SC. ${ }^{2,3(A, B, C, D, E)}$ \\ daniel.rojas.valverde@una.cr \\ ${ }^{2}$ Centro de Investigación y Diagnóstico en Salud y Deporte, Universidad Nacional, \\ Costa Rica \\ ${ }^{3}$ Escuela Ciencias del Movimiento Humano y Calidad de Vida, \\ Universidad Nacional, Costa Rica
}

Envío original: 10/08/2016; reenviado: 16/11/2016, 03/03/217, 26/05/2017;

aceptado: 26/05/2017; publicado 28/06/2017.

Doi: http://dx.doi.org/10.15517/pensarmov.v15i1.29640

\section{RESUMEN}

Rojas-Valverde, D., Morera-Castro, M., Montoya-Rodríguez, J., \& Gutiérrez-Vargas, R. (2017). Demandas cinemáticas en dos tipos de espacios reducidos en jugadores universitarios de fútbol de Costa Rica. Pensar en Movimiento: Revista de Ciencias del Ejercicio y la Salud, 15(1), 12-22. El propósito de esta investigación fue comparar las demandas cinemáticas en dos tipos de dimensiones de juego en 14 jugadores costarricenses de fútbol universitario. Estos jugaron dos juegos en campo reducido (SSG) (2x10 min, $3 \mathrm{~min}$ de descanso): C1 $\left(600 \mathrm{~m}^{2}\right)$ y C2 $\left(1200 \mathrm{~m}^{2}\right)$. Se utilizó un Sistema de Posicionamiento Global para medir las variables cinemáticas y fisiológicas en ambas condiciones. Se utilizó un ANOVA mixto; los resultados sugirieron que C1 y C2 fueron estadísticamente diferentes en velocidad $(\mathrm{C} 1<\mathrm{C} 2)$, distancia $(\mathrm{C} 1<\mathrm{C} 2)$ y frecuencia cardíaca (C1> C2). Al analizar la distancia recorrida por la categoría de velocidad, en acciones de carrera baja, moderada y alta intensidad, los resultados sugieren que C2 posee mayores intensidades en comparación con C1. Esta investigación confirma los resultados de estudios previos sobre la efectividad de SSG para simular condiciones de juego real en

\footnotetext{
${ }^{1}$ Versión traducida al español. También está disponible en la versión original en inglés en esta revista.
} 
períodos cortos de tiempo. Conclusiones: El C2 presentó una mayor intensidad en comparación con el juego C1, con menores demandas fisiológicas. Asimismo, el C2 refleja de manera más exacta las condiciones en partidos oficiales de jugadores costarricenses.

Palabras clave: Sistemas de Posicionamiento Global (GPS); jóvenes; deportes; velocidad; distancia.

El fútbol es probablemente el deporte más popular del mundo, con millones de jugadores activos a nivel mundial. Como lo explican Jones y Drust (2007), el rendimiento en el fútbol es el resultado de las capacidades fisiológicas y psicológicas de los jugadores, factores sociales y habilidades técnicas y tácticas. Actualmente, este juego demanda altas intensidades, con el fin de ejecutar un rendimiento dinámico y rápido (Safania, Alizadeh \& Nourshahi, 2011). Las demandas exigidas en este deporte conllevan a que los esfuerzos de alta intensidad se entremezclan con periodos de carga de baja intensidad (Romero \& Fernández, 2014), requiriendo entonces que el personal profesional de campo mejore sus metodologías durante el entrenamiento, con el propósito de lograr adaptaciones fisiológicas y de desempeño requeridos para los cambios en la dinámica del juego.

Debido a la gran influencia de esta disciplina, la tecnología se ha convertido en una herramienta esencial para mejorar la calidad y la eficiencia del juego. Avances recientes en los recursos tecnológicos permiten mantener mejores controles en el entrenamiento y obtener planificaciones y prescripciones más rigurosas y precisas en las cargas de entrenamiento (Barbero, Vera \& Castagna, 2006; Casamichana \& Castellano, 2010).

Actualmente, la tecnología, por ejemplo, monitores de ritmo cardíaco, analizadores de lactato, Sistemas de Posicionamiento Global (GPS), monitores de frecuencia respiratoria, termómetros de monitorización remota, análisis de video, entre otros dispositivos, han facilitado las mediciones, evaluaciones y seguimiento de las demandas fisiológicas en deportes como el fútbol (Barbero, Barbero, Gómez \& Castagna, 2009, Casamichana \& Castellano, 2010, Hill-Haas, Dawson, Impellizzeri \& Coutts, 2011). Estos dispositivos permiten un mejor control en el entrenamiento y la ejecución de los jugadores, ya sea durante la práctica o en los juegos oficiales.

Daneshjoo, Halim, Rahnama y Yusof $(\underline{2013})$ y Stolen, Chamari, Castagna y Wisloff (2005) mencionan que estos avances tecnológicos permiten detectar cambios como variaciones en la intensidad del juego. En el fútbol se combinan varias acciones intermitentes de alta intensidad y de corta duración y ejercicios de base aeróbica, lo que repercute en las características morfológicas y mecánicas de los miembros inferiores, tales como mayor rigidez y tono muscular (Kubo, Kanehisa, Ito \& Fukunaga, 2001).

En el fútbol, los juegos con dimensiones reducidas (SSG) se utilizan como un método para lograr altas intensidades, simulando las características de un juego oficial (Aguilar, Botelho, Lago, Macas, \& Sampaio, 2012; Casamichana, Castellano, González, García \& García, 2011; Katis \& Kellis, 2009; Safania et al., 2011). Esta actividad de entrenamiento es una adaptación de la situación del juego real aplicada en la práctica, variando las dimensiones del terreno de juego, reduciendo el número de jugadores participantes y 
modificando las reglas oficiales, como la participación del portero y la duración del trabajo físico (Casamichana \& Castellano, 2010; Casamichana \& Castellano, 2011; Dellal et al., 2012; Hill-Haas, et al., 2011; Jones \& Drust, 2007; Katis \& Kellis, 2009; Safania et al., 2011).

Los SSG son un método simple, aunque altamente efectivo y eficiente. Los resultados de los estudios sugieren que esta metodología aumenta significativamente la capacidad fisiológica del jugador. Principalmente, busca traducir la complejidad de la situación de juego a las sesiones de entrenamiento, a través de una mejora holística, donde más allá de los avances físicos y técnicos y tácticos, se debe utilizar una toma de decisiones rápida y eficiente (Dellal et al., 2012).

El uso del GPS ha sido incorporado para controlar las sesiones de entrenamiento, para cuantificar las cargas físicas y fisiológicas que un ejercicio físico causa, a través de variables como la frecuencia cardiaca, la velocidad media, los impactos y la distancia total cubierta por un jugador, permitiendo diariamente, semanalmente o por sesión, la cuantificación del volumen de la estación, para grupos e individuos, en tiempo real (Gómez, Pallarés, Díaz \& Bradley, 2013).

Debido a la necesidad que poseen los preparadores físicos y entrenadores de fútbol de controlar la intensidad del entrenamiento y la competencia, es esencial realizar estudios que se enfoquen en determinar las variaciones de intensidad dependiendo de las dimensiones de terreno. El propósito de este estudio fue comparar las demandas cinemáticas en dos dimensiones de terreno en jugadores de fútbol universitario de Costa Rica.

\section{METODOLOGÍA}

Participantes. Participaron un total de 14 jugadores masculinos (edad $20.9 \pm 1.92$ años, peso $69.6 \pm 7.3 \mathrm{~kg}$, altura $172 \mathrm{~cm} \pm 6.3$ ). Estos jugadores estaban entrenando de 3 a 4 veces por semana y competían regularmente, al menos una vez a la semana. Pertenecen a un equipo de fútbol universitario de Costa Rica, el cual se encontraba en proceso de preparación para la fase eliminatoria entre universidades, en el 2015.

Todos los sujetos fueron informados de los detalles de los procedimientos experimentales, los riesgos asociados y las incomodidades. Cada sujeto dio su consentimiento informado por escrito, según los criterios de la Declaración de Helsinki sobre la investigación biomédica en seres humanos (18 ${ }^{\text {a }}$ Asamblea Médica, 1964, revisada en Fortaleza en 2013).

Instrumentos. La medición del peso corporal se realizó utilizando una escala digital (sensibilidad $\pm 0.1 \mathrm{~kg}$ ) (Serie Elite BC554, Tanita-Ironman, Illinois, Estados Unidos) y para conocer la altura de los jugadores se utilizó un estadiómetro de pared.

Para medir las variables cinemáticas como velocidad y distancia, se utilizó un Sistema de Posicionamiento Global (GPS) (SP PRO X II GPSports®, 15Hz, Canberra, Australia). La validez y fiabilidad de los dispositivos GPS de $15 \mathrm{~Hz}$ han sido demostradas por Barbero et al. (2009). Según los autores, este equipo tiene una alta correlación en pruebas de velocidad y sprint $\left(r^{2}=0.87, p<0.001 ; r^{2}=0.94, p<0.001\right)$. Asimismo, la velocidad máxima 
acumulada y la máxima alcanzaron un bajo coeficiente de variación (CV $=1.7 \%$ y $1.2 \%$ respectivamente). A su vez, Johnston, Watsford, Kelly, Pine y Spurrs (2014) reportan confiabilidad aceptable a través de una prueba temporal (test, re-test, $r=.75$ ). Este instrumento se utilizó para cuantificar las variables: promedio de la distancia en metros cubiertos por minuto $(\mathrm{m} / \mathrm{min})$, frecuencia cardíaca media (latidos $/ \mathrm{min}$ ) y velocidad media (km / h). Se utilizó el software Team AMS®), V2.5.4 (GPSports, Camberra, Australia) para el análisis de la información.

Para realizar el análisis de la información recogida por GPS, la distancia recorrida $(\mathrm{m})$ se clasificó por intensidad de movimiento, según Di Salvo et al. (2007), en cuatro categorías: caminando y trotando $(0-11 \mathrm{~km} / \mathrm{h})$, porcentaje de carrera a baja intensidad (11.1-14 km / h), porcentaje de carrera a moderada intensidad (14.1-19 km / h) y porcentaje de carrera a alta intensidad (19.1- $23 \mathrm{~km} / \mathrm{h})$.

Procedimientos. Se realizó una sesión informativa con los participantes y se firmó el consentimiento informado. Además, se midió el peso y la altura de cada uno.

Se midieron en días consecutivos dos condiciones de espacios reducidos, con una diferencia de 24 horas, y se realizaron antes del entrenamiento normal (7:00 am) y con una activación/calentamiento 10 minutos antes de C1 y C2. La condición 1 (C1) posee una dimensión de 20×30 metros $\left(600 \mathrm{~m}^{2}\right.$ de área de lanzamiento total $-42.86 \mathrm{~m}^{2}$ de área por jugador [APP]) y la condición 2 (C2) posee 30x40 metros (1200 $\mathrm{m}^{2}$ de área de lanzamiento total - $85.1 \mathrm{~m}^{2} \mathrm{APP}$ ). Ambas sesiones se realizaron en el mismo terreno (césped natural), con los mismos jugadores (7vs7, dos equipos seleccionados al azar y mantenidos en ambas condiciones), los mismos balones y el mismo tiempo total de juego (2x10 min, con 3 minutos de reposo e hidratación). Las líneas de goles eran de tres metros de ancho, no había porteros, y había dos balones en cada lado del campo para mantener la dinámica del juego, reduciendo las pausas. Se pidió a los jugadores que no realizaran ejercicio extenuante al menos 24 horas antes de realizar ambas condiciones.

Análisis estadístico. Los resultados se presentan por medio de promedios \pm desviación estándar $(D E)$. Los datos se analizaron para la distribución normal usando la prueba de Shapiro-Wilk. Los datos de la frecuencia cardiaca, la distancia, la velocidad y el porcentaje de distancia recorrida en las intensidades en carrera estática, baja, moderada y alta intensidad se sometieron a un ANOVA de modelo mixto de 2 (condición) x 2 (período), con un alfa previa de $p<.05$. Las magnitudes de las diferencias para todas las variables se analizaron usando el cuadrado parcial omega $\left(\omega_{p}{ }^{2}\right)$ para el análisis de ANOVA, categorización cualitativa como $\omega_{p}^{2}=.15$ efecto alto, $\omega_{p}^{2}=.06$ efecto moderado y $\omega_{p}^{2}=.01$ como efecto pequeño (Cohen, 1977), este análisis se implementó porque $\omega_{p}^{2}$ no se ve afectado en muestras de tamaño pequeño (Moncada, Solera \& Salazar, 2002). El análisis de los datos se realizó utilizando Paquete Estadístico para las Ciencias Sociales (SPSS, IBM, SPSS Statistics, V 22.0 Chicago, IL, EE.UU.). 


\section{RESULTADOS}

En la Tabla 1 y 2 se presenta la estadística descriptiva de las variables en estudio según periodo y condición.

Tabla 1

Estadística descriptiva de las variables distancia, velocidad y frecuencia cardíaca según período y condición

\begin{tabular}{cccc}
\hline Variable & Período & $\mathbf{C 1}\left(\mathbf{6 0 0} \mathbf{~ m}^{\mathbf{2}}\right)$ & $\mathbf{C 2}\left(\mathbf{1 2 0 0} \mathbf{~}^{\mathbf{2}}\right)$ \\
\hline \multirow{2}{*}{ Distancia $(\mathrm{m} / \mathrm{min})$} & Primero & $107.4 \pm 8.3$ & $115.7 \pm 7.4$ \\
& Segundo & $105.9 \pm 9$ & $116.3 \pm 10.5$ \\
\hline \multirow{2}{*}{ Velocidad $(\mathrm{km} / \mathrm{h})$} & Primero & $6.4 \pm .5$ & $6.9 \pm .4$ \\
& Segundo & $6.4 \pm .5$ & $7 \pm .6$ \\
\hline Frecuencia cardiaca & Primero & $163.3 \pm 9.2$ & $152.1 \pm 11.3$ \\
(bpm) & Segundo & $166 \pm 10.2$ & $155.1 \pm 18.6$ \\
\hline
\end{tabular}

Fuente: elaboración propia.

Tabla 2

Estadística descriptiva de la distancia recorrida $(m)$ según la intensidad en período y la condición

\begin{tabular}{lccc}
\hline \multicolumn{1}{c}{ Variable } & Período & $\mathbf{C 1}\left(\mathbf{6 0 0} \mathbf{~ m}^{\mathbf{2}}\right)$ & $\mathbf{C 2}\left(\mathbf{1 2 0 0} \mathbf{~}^{\mathbf{2}}\right)$ \\
\hline Caminando y & Primero & $848 \pm 47.5$ & $816.2 \pm 65.3$ \\
trotando $(\mathrm{m})$ & Segundo & $826.5 \pm 56.5$ & $819 \pm 64.68$ \\
\hline Carrera a intensidad & Primero & $152.1 \pm 38.5$ & $198 \pm 24.6$ \\
baja $(\mathrm{m})$ & Segundo & $145.6 \pm 32.9$ & $193.6 \pm 50.1$ \\
\hline Carrera a intensidad & Primero & $70.4 \pm 28.7$ & $121.9 \pm 42.4$ \\
moderada $(\mathrm{m})$ & Segundo & $75.6 \pm 14.6$ & $121.4 \pm 50.2$ \\
\hline Carrera a intensidad & Primero & $3.9 \pm 5$ & $17.9 \pm 14.4$ \\
$\quad$ alta $(\mathrm{m})$ & Segundo & $9.7 \pm 9.2$ & $23.9 \pm 25.9$ \\
\hline
\end{tabular}

Fuente: elaboración propia.

El ANOVA de modelo mixto 2x2 no mostró interacción estadísticamente significativa (condición vs período) en distancia, $F_{(1,13)}=.366, p=.551, \omega_{p}^{2}=0$ (bajo). Sin embargo, se encontró un efecto principal significativo según la condición $F_{(1,13)}=11.833, p=.002, \omega_{p}^{2}=$ 0.27 (alto), siendo C2 mayor que $\mathrm{C} 1$ en la distancia; no se encontró diferencia significativa en el análisis principal según el período $F_{(1,13)}=.068, p=.796, \omega_{p}^{2}=0$ (bajo).

EI ANOVA no mostró interacción estadísticamente significativa (condición vs período) en velocidad, $F_{(1,13)=} .380, p=.543, \omega_{p}^{2}=0$ (bajo). Sin embargo, se encontró un efecto principal significativo según la condición $F_{(1,13)}=11.833, p=.002, \omega_{p}^{2}=0.27$ (alto), siendo C2 
mayor que $\mathrm{C} 1$ en velocidad; no hubo diferencia significativa en el análisis principal según el período $F_{(1,13)}=.026, p=.872, \omega_{p}^{2}=0$ (bajo).

Los resultados no demostraron interacción estadísticamente significativa (condición vs período) en la frecuencia cardíaca, $F_{(1,13)}=.009, p=.924, \omega_{p}^{2}=0$ (bajo). Sin embargo, hubo un efecto principal significativo según la condición $F_{(1,13)}=6.048, p=.021, \omega_{p}^{2}=.15$ (alto), siendo C1 mayor que C2 en la frecuencia cardíaca; no se encontró diferencia significativa en el análisis principal según el período $F_{(1,13)=} .2 .418 p=.132, \omega_{p}^{2}=.04$ (bajo).

EI ANOVA modelo mixto 2x2 para la distancia recorrida con base en la intensidad, no mostró interacción estadística en la categoría caminando y trotando, $F_{(1,26)}=.313, p=.581$, $\omega_{p}^{2}=0$ (bajo); carrera a baja intensidad, $F_{(1,26)}=.015, p=.904, \omega_{p}^{2}=0$ (bajo); carrera a intensidad moderada, $F_{(1,26)}=.537, p=.470, \omega_{p}^{2}=0$ (bajo); carrera a alta intensidad, $F_{(1,26)}=$ $.052, p=.822, \omega_{p}^{2}=0$ (bajo). El análisis de los efectos principales según la condición de estas variables cinemáticas indicó diferencias significativas en: caminando y trotando, $F_{(1,26)}=23.639, p=.000, \omega_{p}^{2}=.45$ (alto); carrera a baja intensidad, $F_{(1,26)}=7.009, p=.014$, $\omega_{p}^{2}=.18$ (alto); carrera a intensidad moderada, $F_{(1,26)}=15.82, p=.000, \omega_{p}^{2}=.35$ (alto); carrera a alta intensidad, $F_{(1,26)}=13.871, p=.001, \omega_{p}^{2}=.31$ (alto).

El análisis de los efectos principales según el período de la distancia recorrida con base en la intensidad indicó que no hay diferencias significativas en: caminando y trotando, $F_{(1,26)}=1.469, p=.236, \omega_{p}^{2}=.02$ (bajo); carrera a Baja intensidad, $F_{(1,26)}=.387, p=.539, \omega_{p}^{2}=$ 0 (bajo); carrera a intensidad moderada, $F_{(1,26)}=.09, p=.766, \omega_{p}^{2}=0$ (bajo); carrera a alta intensidad, $F_{(1,26)}=2.888, p=.101, \omega_{p}^{2}=.06$ (moderado).

\section{DISCUSIÓN}

Se sabe que en la actualidad las metodologías de formación han favorecido trabajos de alta intensidad en condiciones que se asemejen a la competencia. Los juegos en dimensiones reducidas han llegado a cumplir con estos requisitos de intensidad (Romero \& Fernández, 2014). Debido a que hay múltiples interacciones, el análisis se hace sobre la base del espacio en metros cuadrados que cada jugador tiene que correr en el juego (Febré et al., 2015).

La edad, la talla y el peso de los participantes en este estudio son similares a otros: Castellano, Fernández, Castillo y Casamichana (2010), con una muestra de jóvenes universitarios $(20.1 \pm 1.2$ años, altura $176.3 \pm 9.9 \mathrm{~cm}$ y peso $63.5 \pm 8.4 \mathrm{~kg})$ y Vargas, Urkiza y Gil (2015), que estudiaron el comportamiento de diferentes variables en jugadores con datos antropométricos similares $(20.9 \pm 1.7$ años, $1.80 \pm 0.05 \mathrm{~cm}, 73.1 \pm 5.3 \mathrm{~kg})$ durante el desempeño de SSG en entrenamiento y partidos.

Los resultados de este estudio demostraron que un aumento de las dimensiones del terreno conduce a condiciones físicas más exigentes; por ejemplo, comparando $\mathrm{C} 1 \mathrm{con} \mathrm{C} 2$, este último tiene diferencias significativamente mayores que $\mathrm{C} 1$ en la distancia recorrida en el primero y segundo período. Los resultados de los metros recorridos por minuto en $\mathrm{C} 1$ y C2 son semejantes a los obtenidos por otros estudios de SSG en condiciones similares al presente estudio (Casamichana, San Ramón-Quintana, Castellano \& Calleja-González, 
2012, Casamichana \& Castellano, 2010, Casamichana, Castellano \& Hernández-Mendo, 2014; Hill-Haas, Rowsell, Dawson \& Coutts, 2009; Rampinini et al., 2007).

En cuanto a la velocidad media, hubo diferencias significativas entre $\mathrm{C} 1$ y C2, en el primer período y segundo período, siendo $\mathrm{C} 2$ significativamente mayor que $\mathrm{C} 1$. Estudios anteriores mencionan que, a medida que aumentan las dimensiones del terreno, los requerimientos cinemáticos (velocidad y distancia promedio) aumentan proporcionalmente (Casamichana, Castellano \& Castagna, 2012, Dellal et al., 2012, Nevado-Garrosa \& Suárez-Arrones, 2015; Rampinini et al., 2007).

Los resultados de este estudio sugieren diferencias significativas más altas en las categorías de velocidad según la condición, siendo C2 mayor en todas las categorías, excepto en la categoría caminando y trotando. Esto significa que hubo acciones de mayor intensidad en C2, lo cual queda claro cuando se observan las diferencias significativas entre las condiciones $\mathrm{C} 1$ y $\mathrm{C} 2$, no solo en las categorías de carrera a baja intensidad, sino en aquellas que requieren alta intensidad y velocidad, similares a las reportadas previamente por Nevado-Garrosa y Suárez-Arrones (2015), Casamichana, San Ramón-Quintana, Calleja-González y Castellano (2013) y Dellal et al., (2012).

Analizando la efectividad de SSG para simular condiciones reales de juego en jugadores de fútbol costarricenses, se afirma que tanto el $\mathrm{C} 1$ como $\mathrm{C} 2$ simulan las demandas cinemáticas y las demandas de frecuencia cardiaca de los partidos oficiales reportados previamente en un estudio con una muestra de jugadores brasileños (Barros, et 2007) y los jugadores costarricenses (Gutiérrez-Vargas et al., 2015), y el análisis de los jugadores de la Copa Mundial de Sudáfrica 2010 (Clemente, Santos, Lourenco, Ognyanova \& Mendes, 2013). Por lo tanto, la distribución porcentual de la distancia recorrida por intensidad de velocidad fue similar a los estudios previos realizados en 11 vs 11 (Barros et al., 2007 y Gutiérrez-Vargas et al., 2015), lo que indica que el mayor porcentaje de distancia en la dimensión de terreno oficial se ejecuta a la velocidad de detenido-trotando.

En cuanto a los datos fisiológicos, se obtuvieron diferencias significativamente mayores en C1 en comparación con C2, similares a Casamichana et al. (2014) y Casamichana et al. (2011). Estos resultados están en contraposición con los mencionados por Kelly y Drust (2009), quienes indicaron que la frecuencia cardiaca no varió significativamente al ejecutar SSG en diferentes dimensiones de terreno $\left(600 \mathrm{~m}^{2}, 1200 \mathrm{~m}^{2}\right.$ y $2500 \mathrm{~m}^{2}$ ). Según Allen, Butterfley, Welsh y Wood (1998) significan que la frecuencia cardiaca en un terreno menor es mayor porque hay una mayor participación de los jugadores en la actividad en comparación con dimensiones de terreno más grandes. Febré et al. (2015) señalaron que la frecuencia cardíaca era significativamente menor cuando se jugaba en una APP más pequeña $\left(90 \mathrm{~m}^{2}\right)$ que cuando se jugaba en una más grande (150 $\mathrm{m}^{2}$ por jugador), contrariamente al presente estudio, donde la APP más pequeña $\left(42,86 \mathrm{~m}^{2}\right)$ arrojó un promedio de la frecuencia cardíaca mayor que la APP más grande $\left(85.71 \mathrm{~m}^{2}\right)$.

Estos resultados, relacionados con el comportamiento de las variables cinemáticas en SSG son relevantes debido al uso extendido de este tipo de métodos de entrenamiento para equipos de fútbol en Costa Rica y en todo el mundo, lo que proporciona más información a los entrenadores y al personal respecto a qué tipo de variables cambiar para obtener respuestas más altas o más bajas en velocidad, distancia o ritmo cardíaco. 
Esta investigación confirma los hallazgos de estudios previos sobre la efectividad de SSG para simular condiciones reales de juego en cortos períodos de tiempo. Los resultados indicaron que C2 tenía mayores intensidades en comparación con el juego C1, con menor demanda fisiológica. Asimismo, el C2 se parece más a partidos en condiciones oficiales del jugador costarricense.

\section{REFERENCIAS}

Aguilar, M., Botelho, G., Lago, C., Macas, V., \& Sampaio, J. (2012). A review on the effects of soccer small-sided games. Journal of Human Kinetics, 33, 103-113. Recuperado de http://www.johk.pl/volume 33.html

Allen, J.D., Butterfly, R., Welsh, M.A., \& Wood, R. (1998). The physical and physio-logical value of 5-a-side soccer training to 11-a-side match play. Journal of Human Movement Studies, 34(1), 1-19. Recuperado de https://www.researchgate.net/publication/ 224767111 The Physical and Physiological Value of 5-A-Side Soccer Training to 11-A-Side Match Play

Barbero, J., Vera, J., \& Castagna, C. (2006). Cuantificación de la carga de fútbol: Análisis de un juego en Espacio Reducido. Journal PubliCE Premium. Recuperado de http://gse.com/es/evaluacion-deportiva/articulos/cuantificacion-de-la-carga-en-futbolanalisis-de-un-juego-en-espacio-reducido-783

Barbero, J., Barbero, V., Gómez, M., \& Castagna, C. (2009). Análisis cinemático del perfil de actividad en jugadoras infantiles de fútbol mediante tecnología GPS. Kronos Rendimiento en el deporte, 18(14), 35-42. Recuperado de http://abacus. universidadeuropea.es/handle/11268/3257

Barros, R., Misuta, M., Menezes, R., Figueroa, P., Moura, F., Cunha, S., Anido, R., \& Leite, R. (2007). Analysis of distance covered by first division Brazilian soccer players obtained with an automatic tracking method. Journal of Sport Science and Medicine, 6, 233-242. Recuperado de http://www.jssm.org/abstresearcha.php?id=jssm-06233.xml

Casamichana, D. \& Castellano, J. (2010). Time-motion, heart rate, perceptual and motor behavior demands in small-sides soccer games: Effects of pitch size. Journal of Sports Sciences, 28(14), 1615-1623. doi: https://doi.org/10.1080/02640414.2010.521168

Casamichana, D., Castellano, J., González, A., García. H., \& García., J. (2011). Demanda fisiológica en juegos reducidos de fútbol con diferente orientación del espacio. International Journal of Sport Science, 23(7), 141-154. Recuperado de http://www.cafyd.com/REVISTA/02306.pdf

Casamichana, D. \& Castellano, J. (2011). Demandas físicas en jugadores semiprofesionales de fútbol: ¿Se entrena igual que se compite? Revista Cultura Ciencia Deporte, 6(17), 121-127. Recuperado de http://www.redalyc.org.una.idm. oclc. org/articulo.oa?id=163022532006.

Casamichana, D., Castellano, J., \& Castagna, C. (2012). Comparing the Physical Demands of Friendly Matches and Small-Sided Games in Semiprofessional Soccer Players. 
Journal of Strength and Conditioning Research, 26(3), 837-843. Doi: https://doi.org/10.1519/JSC.0b013e31822a61cf

Casamichana, D., Castellano, J., \& Hernández-Mendo, A. (2014). La Teoría de la generalizabilidad aplicada al estudio del perfil físico durante juegos reducidos con diferente orientación del espacio en fútbol. International Journal of Sport Science, 10(37), 194-205. doi: http://dx.doi.org/10.5232/ricyde2014.03702

Casamichana, D., San Ramón-Quintana, J., Calleja-González, J., \& Castellano, J. (2013). Utilización de la limitación de contactos en el entrenamiento en fútbol: ¿ afecta a las demandas físicas y fisiológicas?. International Journal of Sport Science, (9)33, 208221. doi: https://doi.org/10.5232/ricyde2013.03301

Casamichana, D., San Ramón-Quintana, J., Castellano, J., \& Calleja-González, J. (2012). Demandas físicas y fisiológicas en jugadores absolutos no profesionales durante partidos de fútbol 7: un estudio de caso. Cultura Ciencia Deporte, 7(20), 115-123. Recuperado de http://ccd.ucam.edu/index.php/revista/article/view/57

Castellano, J., Fernández, J., Castillo, A., \& Casamichana, D. (2010). Fiabilidad intraparticipante de diferentes modelos de dispositivos GPS implementados en un partido de Fútbol 7. Cultura Ciencia Deporte, 5(14), 85-95. Recuperado de http://ccd.ucam.edu/index.php/revista/article/view/97

Clemente, F., Santos, M., Lourenco, F., Ognyanova, M., \& Mendez, R. (2013). Activity Profiles of Soccer Players during the 2010 Word Cup. Journal of Human Kinetics, 38 , 201-211. Recuperado de http://www.johk.pl/files/johk-vol38-2013-21.pdf

Cohen, J. (1977). Statistical power analysis for the behavioral sciences. Recuperado de https://books.google.co.cr/books/about/Statistical power analysis for the behav.ht ml?id=AG1qAAAAMAAJ\&redir esc $=y$

Daneshjoo, A., Halim, A., Rahnama, N., \& Yusof, A. (2013). The effects of injury prevention warm-up programmes oh knee strength in male soccer players. Biology of Sport, 30(4), 281-288. doi: https://doi.org/10.5604/20831862.1077554

Dellal, A., Owenc, A., Wong, D.P., Krustrup, P., Van Exsel, M., \& Mallo, J. (2012). Technical and physical demands of small vs. large sided games in relation to playing position in elite soccer. Human Movement Science, 31(4), 957-969. doi: https://doi.org/10.1016/j.humov.2011.08.013

Di Salvo, V., Baron, R., Tschan, H., Calderón, FJ., Bachl, N., \& Pigozzi, F. (2007). Performance characteristics according to playing position in elite soccer. International Journal of Sports Medicine, 28(3), 222-7. Recuperado de https://www.researchgate.net/publication/6769686 Performance Characteristics Ac cording to Playing Position in Elite Soccer

Febré, R., Chirosa, J., Casamichana, D., Chirosa, Martín-Tamayo, I., \& Pablos, C. (2015). Influencia de la densidad de jugadores sobre la frecuencia cardiaca y respuestas técnicas en jóvenes jugadores de fútbol. Revista Internacional de Ciencias del Deporte, 11(40). Recuperado de http://www.cafyd.com/REVISTA/ojs/index. php/ricyde/article/view/731

Gómez, A. J., Pallarés, J., Díaz, A., \& Bradley, P. (2013). Cuantificación de la carga física y psicológica en fútbol profesional: diferencias según el nivel competitivo y efectos -20 - 
sobre el resultado en competición oficial. Revista de Psicología del deporte, 22(2), 463-469. Recuperado de http://www.rpd-online.com/article/view/v22-n2-gomez-diazpallares-diaz-bradley

Gutiérrez-Vargas, R., Rojas-Valverde, D., Jiménez-Madrigal, E., Sánchez-Ureña, B., SalasNaranjo, A., Gutiérrez-Vargas J.C., \& Salazar-Cruz, I. (2015). Parámetros Cinemáticos y Técnicos en Jugadores Jóvenes de fútbol Después de Modificar la Regla del Fuera de Juego (Regla 11). Kronos, 14(2). Recuperado de http://gse.com/es/entrenamiento-en-futbol/articulos/parametros-cinematicos-y-tecnicos-enjugadores-jovenes-de-futbol-despues-de-modificar-la-regla-del-fuera-de-juego-regla11-1905

Hill-Haas, S., Dawson, B., Impellizzeri, F., \& Coutts, A. (2011). Physiology of small sided games training in football. Sports Medicine, 41(3), 199-220. doi: https://doi.org/10.2165/11539740-000000000-00000

Hill-Haas, S., Rowsell, G., Dawson, B., \& Coutts, A. J. (2009). Acute physiological responses and time-motion characteristics of two small-sided training regimes in youth soccer players. The Journal of Strength \& Conditioning Research, 23(1), 111-115. doi: https://doi.org/10.1519/JSC.0b013e31818efc1a

Johnston, R., Watsford, M., Kelly, S., Matthew, P., \& Spurrs, R. (2014). The validity and reliability of $10 \mathrm{~Hz}$ and $15 \mathrm{hz}$ GPs units for assessing athlete movement demands. Journal of Strength and Conditioning Research, 28(6),1649-1655. doi: https://doi.org/10.1519/JSC.0000000000000323

Jones, S. \& Drust, B. (2007). Physiological and technical demands of 4 v 4 and 8 v 8 games in elite youth soccer player. Kinesiology, 39(2), 150-156. Recuperado de https:// pdfs.semanticscholar.org/e85e/6700cb62c0b3630c8f8e52396cc33baf301b.pdf

Katis, A. \& Kellis, E. (2009). Effects of small-sided games on physical conditioning and performance in young soccer players. Journal of Sports Science and Medicine, 8(3), 374-380. Recuperado de http://www.jssm.org/researchjssm-08-374.xml.xml

Kelly, D. \& Drust, B. (2009). The effect of pitch dimensions on heart rate responses and technical demands of small-sided soccer games in elite players. Journal of Science and Medicine in Sport, 12(4), 475-479. doi: https://doi.org/10.1016/ j.jsams.2008.01.010

Kubo, K., Kanehisa, H., Ito, M., \& Fukunaha T. (2001). Effects of isometric training on the elasticity of human tendon structures in vivo. Journal of Applied Physiology. 91(1), 2632. Recuperado de https://www.ncbi.nlm.nih.gov/pubmed/11408409

Moncada, J., Solera, A., \& Salazar, W. (2002). Fuentes de varianza e indices de varianza explicada en las ciencias del movimiento humano. Pensar en Movimiento: Revista de Ciencias del Ejercicio y la Salud, 2 (2): pp 70-74. doi: https://doi.org/ 10.15517/pensarmov.v2i2.398

Nevado-Garrosa, F. \& Suárez-Arrones, L. (2015). Comparación de las demandas físicas de tareas de fútbol reducido y la competición en jugadoras de fútbol sub 13. Cultura Ciencia Deporte, 10 (30), pp. 235-243. Recuperado de http://ccd.ucam.edu/ index.php/revista/article/view/592 
Rampinini, E., Impellizzeri, F., Castagna, C., Abt, G., Chamari, K., Sassi, A., \& Marcora, S. (2007). Factors influencing physiological responses to small-sided soccer games. Journal of Sports Sciences, 25(6), 659-666. doi: https://doi.org/ $10.1080 / 02640410600811858$

Romero, A. \& Fernández, J. (2014). El entrenamiento interválico de alta intensidad (Tesis de Grado). Recuperado de http://dspace.umh.es/bitstream/11000/1911/1/\%C3\%81ngel\% 20Gabriel\%20Romero\%20Ant\%C3\%B3n.pdf

Safania, A., Alizadeh, R., \& Nourshahi, M. (2011). A Comparison of Small- Side Games and Interval Training on Same Selected Physical Fitness Factors in Amateur Soccer Players. Journal of Social Sciences, 7(3), 349-353.doi: https://doi.org/10.3844/ issp.2011.349.353

Stolen, T., Chamari, K., Castagna, C., \& Wisloff, U. (2005). Physiology of Soccer. Sports of Medicine, 35(6), 501-536. doi: https://doi.org/10.2165/00007256-200535060-00004

Vargas, A., Urkiza, I., \& Gil, S. (2015). Efecto de los partidos de pretemporada en la planificación deportiva: Variabilidad en las sesiones de entrenamiento. Retos, 27, 4551. Recuperado de https://recyt.fecyt.es/index.php/retos/article/view/34346

Participación: A- Financiamiento, B- Diseño del estudio, C- Recolección de datos, D- Análisis estadístico e interpretación de resultados, E- Preparación del manuscrito. 